\title{
Esquema de control jerárquico para fijación óptima de consignas en un separador trifásico de un tren de tratamiento de crudo ${ }^{1}$
}

\section{Hierarchical control scheme for optimal setting of setpoints in a three- phase separator of a crude treatment train}

\author{
S.A. Sánchez, C.A. Gaviria
}

Recibido: mayo 20 de 2019 - Aceptado: septiembre 30 de 2019

Resumen-Una de las técnicas para tratar el crudo proveniente de los pozos petroleros es utilizar trenes de tratamiento de crudo para separar las tres fases existentes: gas, petróleo y agua. Uno de los equipos que conforma el tren es el separador trifásico. En este trabajo se demuestra la viabilidad de utilizar un esquema de control jerárquico para la fijación en línea de consignas óptimas de presión y temperatura y así reducir el consumo energético en el tratador térmico. Tal que las variables manipulas y los sets point se encuentren dentro de los límites de la operación segura, con esto se obtienen beneficios económicos y ambientales para esta planta. El logro de esta optimización constituye el aporte principal de este trabajo. Para el desarrollo de este trabajo se diseñó un algoritmo de optimización basado en tres capas jerárquicas en donde la primera capa hace referencia al control directo (PIDs), la segunda capa hace referencia a la optimización de objetivos en estado estacionario (SSTO), la tercera capa hace referencia a la optimización local de objetivos (LSSO). Para la solución a este problema se recurrió a soluciones de optimización lineal y optimización no lineal se relaciona estos algoritmos con el modelo linealizado de la planta, en otros trabajos por lo general se basan en la teoría MPC (Modelo de Control Predictivo). La simulación de los algoritmos se realizó en Matlab y se probaron en una planta simulada en Hysys utilizando el conector Active $\mathrm{x}$ server que conecta los dos softwares. En este experimento se logró un ahorro de un $30 \%$ del consumo energético a su vez se encontró que es posible relacionar el modelo de la planta con los algoritmos de optimización lineal y no lineal con lo cual se concluyó que es viable la implementación de estos algoritmos en este tipo de plantas para la optimización de procesos.

${ }^{1}$ Producto derivado del Proyecto de investigación "Cálculo de consignas óptimas en un tren de tratamiento de crudos pesados" desarrollado en el marco del programa de Maestría en Automática de la Universidad del Cauca.

S. A. Sánchez, Universidad del Cauca, Popayán, Colombia email: sasanchez3@hotmail.com.

C. A. Gaviria, Universidad del Cauca, Popayán, Colombia; e-mail: cgaviria@unicauca.edu.co.

Como citar este artículo: Sánchez, S. A., y Gaviria, C. A. Esquema de control jerárquico para fijación óptima de consignas en un separador trifásico de un tren de tratamiento de crudo, Entre Ciencia e Ingeniería, vol. 13, no. 26, pp. 59-67, julio-diciembre 2019. DOI: https://doi.org/10.31908/19098367.1163.

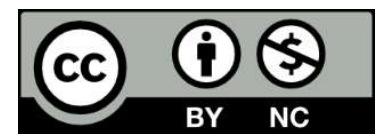

Attribution-NonCommercial 4.0 Intenational (CC By-NC 4.0)
Palabras clave - Separador trifásico; Sistemas jerárquicos; Control Multicapa; Optimización de consignas, Optimización Local de Objetivos, Optimización de Objetivos en estado estacionario, Modelo de Control Predictivo.

\begin{abstract}
- one of the techniques to treat oil from oil wells is to use oil treatment trains to separate the three existing phases: gas, oil, and water. One of the equipments that make up the train is the three-phase separator. This work demonstrates the feasibility of using a hierarchical control scheme for an online setting of optimal pressure and temperature setpoints and thus reducing energy consumption in the heat treater. So that the manipulated variables and the set points are within the limits of the safe operation, with this provides an economic and environmental benefit for this plant. The achievement of this optimization is the main contribution of this work. For the development of this work we designed an optimization algorithm based on three hierarchical layers where the first layer refers to direct control (PIDs), the second layer refers to the optimization of steady state objectives (SSTO), the third layer refers to local optimization of objectives (LSSO). For the solution to this problem, we resorted to solutions of linear optimization and nonlinear optimization these algorithms are related to the linearized model of the plant, in other works are usually based on the theory MPC (Predictive Control Model). The simulation of the algorithms was done in Matlab and tested in a simulated plant in Hysys using the Active $x$ server connector that connects the two software. In this experiment, it was possible to save $30 \%$ of energy consumption. In turn, it was found that it is possible to relate the plant model with the linear and non-linear optimization algorithms, which concluded that the implementation of these algorithms is feasible in this type of plant for process optimization.
\end{abstract}

Keywords - Three-phase separator; Hierarchical systems; Multilayer control; Optimization of setpoint, Local Optimization of Objectives, Optimization of Targets in steady state, Predictive Control Model.

\section{INTRODUCCIÓN}

E n los pozos petroleros se extrae un fluido compuesto principalmente de agua, petróleo crudo y gas, cada una de los cuales usualmente recibe el nombre de fase. Un tren de tratamiento de crudo está compuesto por una serie de equipos de separación cuya finalidad es separar estas tres fases para hacerlas disponibles para el mercado al ser enviadas por sistemas de oleoductos, o para su posterior almacenamiento en 
ambientes adecuados para cada uno. Si las gotas de agua se agrupan y asientan en el fondo de una muestra dentro de un periodo de 3 a 10 minutos, la fracción de agua recibe el nombre de agua libre [1]. Los términos "separador trifásico" y la expresión inglesa "free-water knockout" (FWKO) se utilizan para describir recipientes de presión diseñados para separar y remover el agua libre desde una mezcla de petróleo crudo y agua y hacen uso del principio de separación por efecto de asentamiento por gravedad, donde el petróleo queda en la parte superior mientras que el agua libre queda en el fondo del recipiente. La fase gaseosa se separa en la entrada del separador por evaporación. El término separador trifásico se emplea normalmente cuando hay una gran cantidad de gas a separar desde el líquido, mientras que el término FWKO se emplea en el caso donde la cantidad de gas a separar es pequeña en relación con la cantidad de petróleo y agua.

Un factor importante en el proceso de la fase líquida es el tiempo de retención del líquido en el separador, ya que éste no puede ser demasiado corto tal que la separación por gravedad no tenga efecto, pero tampoco tan grande que permita la acumulación de emulsiones y sedimentos en la interface entre el agua y el petróleo, disminuyendo la eficiencia de separación. Una forma de reducir el tiempo de retención es mediante la agregación ya sea de calor o de químicos para romper emulsiones, o ambas alternativas, en la sección líquida del separador. Cuando se agrega calor, el separador también recibe el nombre en inglés heater-treater y se usa especialmente cuando el crudo llega al separador desde varios pozos y el volumen de crudo a tratar es alto. La fuente de energía calorífica usada por el calentador usualmente es la combustión a gas, pero también puede ser energía eléctrica [2].

Con el fin de maximizar la producción de petróleo y minimizar la cantidad de petróleo residual en el agua, se debe establecer un esquema de control adecuado en el separador. El esquema de control más común utiliza tres compensadores PI de una sola entrada y una sola salida (SISO) donde las variables manipuladas son las aperturas de válvulas de control para los flujos de salida de cada fase, y las variables controladas son la presión, el nivel de agua y el nivel de petróleo [3]. En el caso de tratamiento con calor, se requiere un tercer compensador para mantener la temperatura regulando el flujo de energía calorífica. El trabajo en [4], aborda el problema de las perturbaciones debidas a variaciones de naturaleza oscilatoria en el flujo alimentación del separador, dado que proviene desde los pozos, con velocidades de oscilación que pueden variar entre bajas o altas. Este problema se ha intentado abordar también con esquemas de control MPC no lineal [5].

El trabajo en [18] resalta el problema de ahorro de gas en una planta de tratamiento. Si bien cada lazo de control debe fijar sus consignas dentro de un rango de valores aceptables para la instalación, no es claro si es posible encontrar un conjunto de consignas que, al tiempo que logren un correcto funcionamiento del proceso, también logren un consumo óptimo de la energía calorífica. A la fecha de este trabajo no se han encontrado reportes de aportes en esta dirección. Este problema puede abordarse como uno de diseño de un esquema de control supervisor jerárquico, donde los lazos del proceso, en este caso los de nivel, presión y temperatura, son fijados por un algoritmo de mayor jerarquía, el cual busca la optimización de un criterio de desempeño económico, respetando restricciones, principalmente en cuanto al rango de valores permitidos para las consignas. Tal esquema ha recibido varios nombres en la literatura, tal como estructura de control multicapa [6], u optimización de tiempo real [7].

Dadas las dificultades propias de obtención de datos y de experimentación en un campo petrolero, en este trabajo se utiliza un modelo de simulación de un separador trifásico disponible en el software comercial HYSYS [8], el cual posee modelos de equipos para el campo petrolero, y ha sido reportado como útil en el modelado de separadores trifásicos tanto para tareas de dimensionamiento en régimen permanente como en análisis dinámico [9]. El aporte principal de este trabajo es mostrar, mediante experiencias de simulación en HYSYS, que un enfoque de control jerárquico de dos fases es viable para minimizar el consumo energético de este proceso, con el consecuente potencial beneficio económico en este tipo de plantas.

\section{MARCO TEÓRICO}

La figura Fig. 1 muestra esquemáticamente la construcción de un separador trifásico o FWKO, que usa una fuente de calor como medio para facilitar el rompimiento de la cohesión entre el agua y petróleo. En este equipo se distinguen tres secciones: la frontal, la cual incluye el calentador y un lavador de agua, la cámara de compensación de petróleo, y la sección de coalescencia. Los fluidos ingresan por la sección frontal a través de la entrada de alimentación de emulsión atravesando una cubierta deflectora donde el gas es chispeado y separado. El agua y los sólidos fluyen hacia el fondo por ser más pesados, mientras que el gas y petróleo quedan arriba. El gas libre se rompe y fluye hacia un ecualizador de gas y de allí a la salida de gas. El petróleo, emulsión y agua libre pasa alrededor de la cubierta deflectora a un dispersor ubicado justo debajo de la interface petróleo - agua, donde se "lava" al agua y se separa el agua libre. Para crudos con baja relación gas/petróleo puede requerirse el uso de gas blanquet para mantener la presión, la cual debe mantenerse constante y es la que permite que el flujo de los líquidos por las secciones del tratador. El petróleo y emulsión se calientan conforme suben las paredes vecinas al tubo de fuego y son desnatados en la cámara de compensación de petróleo. Ya que el agua se separa de los fluidos de entrada en la sección frontal, el nivel del agua sube y debe removerse porque de lo contrario sube hasta que desplaza toda la emulsión y comienza a derramarse sobre el vertedero en la sección de compensación de petróleo.

Debe anotarse que, en la mayoría de tratadores de hoy en día, se suele utilizar un intercambiador de calor externo previo al ingreso del fluido en el tratador, con lo que el tamaño del tratador se reduce. Se requiere un control del nivel de petróleo en la sección de compensación para operar la salida de petróleo en condición de tubería llena de líquido. El petróleo y emulsión fluyen a través de un dispersor en la parte posterior de la sección de coalescencia, la cual está llena de fluido. El dispersor distribuye el flujo 
uniformemente a través de la longitud de esta sección. El petróleo tratado sube hacia el colector de petróleo limpio debido a que es más ligero que el agua y la emulsión, donde es colectado y sacado del sistema por la salida de petróleo limpio [10].

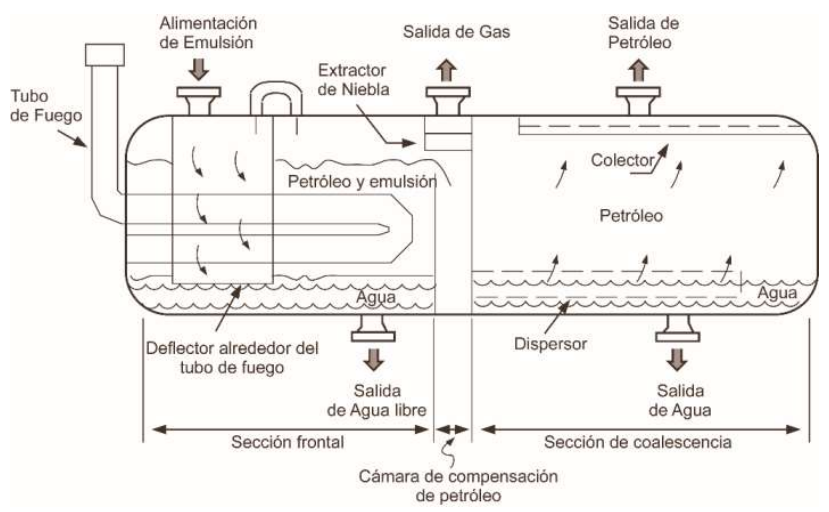

Fig. 1. Esquema simplificado de un separador trifásico con calentador tratador. Tomado de [Crude-Oil].

Para el control del funcionamiento del separador trifásico se suelen utilizar tres lazos de control tipo PI. El controlador PI de nivel de interfaz mantiene la altura de la interface petróleo/agua manipulando una válvula de salida del agua. El nivel del petróleo se controla por un segundo controlador PI a través de una válvula de descarga de petróleo. La presión de la vasija se mantiene constante por el tercer lazo PI a través de una válvula de salida de gas [11]. En el caso del tratador con calor, se requiere además un lazo de control de temperatura, manipulando la fuente de energía del calentador o intercambiador de calor utilizado. En cuanto a la existencia de modelos matemáticos para este tipo de sistemas, los aportes pueden clasificarse según su propósito en dos tipos a saber: modelos para análisis de dimensionamiento y eficiencia del separador, y modelos para el diseño de controladores para el separador.

Los modelos matemáticos utilizados para el análisis de este tipo de equipos se pueden dividir en dos grandes grupos: i) modelos para el dimensionamiento y eficiencia, donde se aproxima el fenómeno del flujo de fluidos polifásicos utilizando ya sea leyes físicas, leyes semiempíricas, o correlaciones netamente empíricas [12], [13], algunos de ellos apoyados en técnicas de correlación sobre experimentos reales $o$ herramientas de análisis computacional de dinámica de fluidos (CFD); y ii) modelos para diseño de controladores, los cuales utilizan asunciones que permiten simplificar las interacciones entre la temperatura y presión con la masa de los componentes y de este modo utilizar los principios de balance de masa y energía comunes en la técnica de control de procesos [14].

El trabajo en [15] utiliza el software HYSYS para investigar propiedades dinámicas y de régimen permanente de un separador trifásico al ser correlacionadas con datos experimentales, concluyendo que es posible utilizar modelos de HYSYS que aproximan adecuadamente el desempeño experimental. HYSYS es un software ampliamente empleado en la industria de ingeniería química para investigación, modelado, desarrollo y diseño. HYSYS proporciona una plataforma para la creación de simulaciones tanto de régimen permanente como dinámicas. Durante el desarrollo del modelo en HYSYS, es esencial usar especificaciones adecuadas de los fluidos y reacciones químicas involucradas. No obstante, especificando solamente parámetros de presión, temperatura, fracción de vapor, rata de flujo másico o molar y la composición del fluido, HYSYS puede generar automáticamente los parámetros restantes de especificación del fluido.

La operación de separación en HYSYS se basa en separación termodinámica perfecta, aunque esta condición no se cumple en el mundo real por la interacción de las tres fases. Sin embargo, HYSYS permite modelar capacidades no ideales de separación tanto en régimen permanente como dinámico, gracias al modelado del fenómeno de arrastre en la fase líquida o gaseosa. Este fenómeno se puede especificar por medio de alguna de tres opciones: basado en la alimentación, basado en el producido, o basado en correlación, donde los datos de alimentación y de producido se correlacionan con base en las leyes físicas que describen este fenómeno.

En cuanto a los esquemas de control, todas las referencias encontradas en este trabajo se han centrado en el problema de la regulación de tres variables controladas: nivel de agua en la interface, nivel de petróleo en la cámara de petróleo y presión en la cámara de gas, a partir de la manipulación de la apertura de válvulas de flujo de salida de agua, petróleo y gas [4], [11], [14], [16], [17]. Dadas las asunciones subyacentes en los modelos simplificados utilizados, en este problema de control se observa un modelo MIMO de tres salidas medidas, con tres entradas manipuladas, donde hay poca o nula interrelación entre la variable de presión con respecto a las variables de nivel, y un acople débil entre las variables de nivel, por lo que se explica que la sintonía de controladores independientes tipo PI es suficiente para lograr el seguimiento de consignas constantes sobre las variables controladas, lo cual se corrobora en la experiencia industrial práctica.

El problema de los disturbios en este sistema de control se ha abordado en trabajos tales como [4], [17], donde las fracciones de masa de petróleo, agua y gas en el flujo de alimentación se consideran como disturbios no medibles del modelo y que deben ser tenidas en cuenta ya que alteran la condición asumida para el régimen de flujo laminar, y llevan a una situación de flujo con oscilaciones entre altas y baja, régimen denominado flujo de lodo, que va en detrimento de la separación produciendo variaciones de flujos y presión en el separador. En [17] se muestra mediante simulación, cómo esas perturbaciones de naturaleza oscilatoria pueden provocar correcciones demasiado fuertes de un controlador tipo PI por la naturaleza integradora de los niveles de petróleo y de agua en la interface, lo que lleva a situaciones de parada de los controladores. En [4] se refieren varios trabajos previos encaminados a la solución del régimen de flujo de lodo, entre ellas, la fijación de dos conjuntos de sintonía de los controladores PI, uno para regímenes de flujo donde se permite un mayor ancho de banda, y otro para condiciones de flujo de lodo donde el ancho debanda se reduce. La estrategia usada en [4] hace uso de tres niveles de control: una capa inferior compuesta de controladores PID esclavos, una intermedia que utiliza un controlador por modelo predictivo no lineal práctico (PNMPC) y un estimador de 
los disturbios, y una superior correspondiente a un control supervisor que define el modo de operación en dependencia de la existencia o no de régimen de flujo de lodo.

En este trabajo se aborda un problema de control diferente al planteado en las referencias previas, donde se investiga si es viable que un esquema de control tenga en cuenta la minimización del consumo energético de la planta, además del logro de las consignas de operación propias del sistema. Se plantea como posible solución a este problema, emplear un esquema de supervisión para la fijación de consignas óptimas.

Un enfoque que ha sido utilizado en plantas químicas y también en el área del petróleo para el problema de fijación de consignas óptimas, es el control jerárquico de dos fases [6]. En este esquema, se distinguen dos fases de control dispuestas en una jerarquía de control supervisor. El nivel inferior está constituido por controladores esclavos tipo PID o MPC, y son los encargados de llevar las variables de proceso a los valores deseados. Cuando deben imponerse restricciones a los valores de las variables manipuladas o a los valores de las variables controladas, la opción más adecuada es el control MPC. Para el nivel superior existen varias variantes propuestas que pueden hacer que este nivel se subdivida en dos capas de control. El objetivo de este nivel superior es establecer cuáles deben ser las consignas para el nivel inferior, teniendo como premisa la optimización de un criterio de desempeño que usualmente obedece a motivaciones económicas. Este problema generalmente lleva a la necesidad de la vigilancia sobre los límites aceptables para la seguridad del proceso, tanto en las variables manipuladas como en las controladas. Así, el diseño de la capa superior se establece en términos de un problema de optimización con restricciones. En [6] se establece que una alternativa simple consiste en fijar un criterio de optimización económica para valores de régimen permanente basados en modelo local (LSSO), teniendo en cuenta restricciones sobre entradas y salidas del modelo de régimen permanente. No obstante, en general no es viable aplicar directamente al nivel inferior estas consignas calculadas, ya que los cambios en las consignas producen cambios dinámicos en las variables de proceso que pueden llevar a comportamientos no deseables en los lazos de bajo nivel. Por tal razón, existe una capa de optimización dinámica en el medio entre la capa LSSO y el nivel jerárquico inferior mediante la utilización de un modelo dinámico predictivo de la planta que tenga en cuenta los efectos de los cambios de consignas en un horizonte de tiempo de predicción y respetando las restricciones sobre las variables controladas y manipuladas. Esta capa dinámica generalmente se implementa en la forma de un control MPC que en [6] se denomina capa MPC-DO. La Fig. 2 ilustra el esquema general de control jerárquico de dos fases.

Ya que el control MPC-DO introduce variaciones dinámicas de las variables, se propone actualizar los valores de optimización de régimen permanente conforme se actualiza la ley de control. Entonces se plantea la necesidad de una capa adicional entre LSSO y la capa MPC-DO, en la cual se actualiza de forma dinámica la consigna óptima de régimen permanente a ser empleada en la capa MPC-DO. Tal capa recibe el nombre de optimización del blanco de régimen permanente (SSTO).

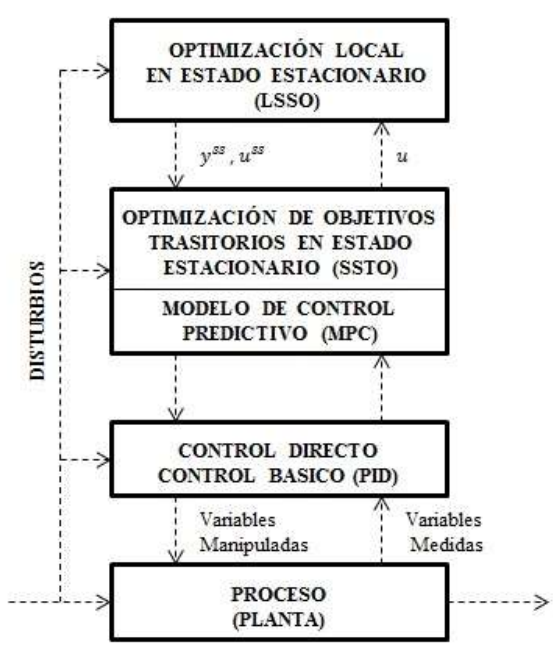

Fig. 2. Esquema general de control jerárquico de dos fases. Fuente: [6].

El problema LSSO se puede establecer formalmente mediante (1).

$$
\begin{gathered}
\min _{u^{s S}, y^{s s}}\left\{J_{E}(k)=c_{u}^{T} u^{s s}-c_{y}^{T} y^{s s}\right\} \\
\text { Sujeto a: } \begin{aligned}
u_{\min } & \leq u^{s s} \leq u_{\max } \\
y_{\min } & \leq y^{s s} \leq y_{\max } \\
y^{s s} & =\boldsymbol{F}\left(u^{s s}, \widetilde{w}\right)
\end{aligned}
\end{gathered}
$$

Donde $J_{E}(k)$ es un criterio de desempeño económico en el instante $k, \boldsymbol{F}\left(u^{s s}, \widetilde{w}\right)$ denota un modelo del proceso en régimen permanente, generalmente un modelo no lineal a menudo dado en una forma numérica implícita, $\widetilde{w}$ es la estimación o medida actual de los disturbios, $c_{u}^{T}$ y $c_{y}^{T}$ son pesos para las consideraciones económicas, $u_{\min }, u_{\max }, y_{\min }$, y $y_{\max }$ son los límites de las restricciones impuestas a las entradas y salidas del proceso. Además, $u$ se refiere a las entradas manipuladas de la planta en tanto que $y$ se refiere a las variables controladas, mientras que $w$ se refiere a los disturbios medibles. La notación $u^{s s}$ se utiliza para expresar los valores de régimen permanente, en este caso de la entrada.

El problema SSTO presenta de manera formal como lo muestra (2).

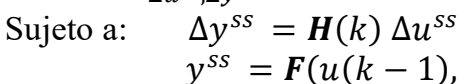

$$
\min _{\Delta u^{s,}, \Delta y^{s s}}\left\{J_{E}(k)=c_{u}^{T} \Delta u^{s s}-c_{y}^{T} \Delta y^{s s}\right\}
$$

$$
\begin{aligned}
& y^{s s}=\boldsymbol{F}(u(k-1), \widehat{w}(k))+\Delta y^{s s}+b \\
& u^{s s}=u(k-1)+\Delta u^{s s} \\
& u_{\min } \leq u^{s s} \leq u_{\max } \\
& y_{\min } \leq y^{s s} \leq y_{\max }
\end{aligned}
$$

Donde $b$ es un desplazamiento constante correspondiente a disturbios no medidos basado en comparación entre las salidas predichas y las medidas. La matriz $\boldsymbol{H}(k)$ es la matriz de ganancia calculada como:

$$
\boldsymbol{H}(k)=\left[\begin{array}{lll}
\frac{\partial \boldsymbol{F}(u(k-1), \widetilde{w}(k))^{T}}{\partial u_{1}} & \cdots & \frac{\partial \boldsymbol{F}(u(k-1), \widetilde{w}(k))^{T}}{\partial u_{n_{u}}}
\end{array}\right]^{T}
$$


Siendo $n_{u}$ el número de entradas. Usualmente $\boldsymbol{H}(k)$ se calcula numéricamente por medio de un método de diferencias finitas.

La optimización dinámica basada en MPC se define en la forma usual como en la ecuación (3), donde $N$ y $N_{u}$ denotan respectivamente los horizontes de control y de predicción, $\left.\boldsymbol{\Delta} \boldsymbol{u}(k)=\left[\Delta u(k \mid k)^{T} \cdots \Delta u\left(k+N_{u}-1 \mid k\right)^{T}\right]^{T}\right)$ es el vector de decisiones de control, $y(k+p \mid k)$ denota la predicción de la salida del proceso para el instante de muestro futuro $k+p$ calculada en el instante de muestreo actual $k$. El objetivo de control es que se llegue al valor deseado de la salida en régimen permanente, $y^{s s}=y^{s p}(k+p \mid k)$, minimizando el criterio de desempeño $J_{M P C}(k)$ al tiempo que se respeten restricciones en el rango de valores de las entradas.

$$
\begin{gathered}
\min _{\Delta u(k)}\left\{J_{M P C}(k)=\sum_{p=1}^{N}\left\|y^{s p}(k+p \mid k)-y(k+p \mid k)\right\|^{2}\right. \\
\left.+\lambda \sum_{p=0}^{N_{u}-1}\|\boldsymbol{\Delta u}(k+p \mid k)\|^{2}\right\} \\
\text { Sujeto a: } \\
u_{\text {min }}^{\leq} \leq u(k+p \mid k) \leq u_{\max }, p=0, \cdots, N_{u}-1 \\
-\boldsymbol{\Delta} \boldsymbol{u}_{\text {max }} \leq \boldsymbol{\Delta u}(k+p \mid k) \leq \boldsymbol{\Delta} \boldsymbol{u}_{\text {max }}, p=0, \cdots, N_{u}-1 \\
y_{\text {min }} \leq y(k+p \mid k) \leq y_{\max }, p=0, \cdots, N
\end{gathered}
$$

\section{METODOLOGIA}

En este trabajo se han fijado como asunciones sobre el separador trifásico: 1) El criterio de optimización económica es el consumo de energía en la sección de intercambio de calor. 2) Las variables controladas son los niveles de la interface, de petróleo, la presión en la vasija y la temperatura en el intercambiador de calor, mientras que las variables manipuladas son las aperturas de las válvulas de salida de agua libre, petróleo, gas y el flujo de energía aplicado al intercambiador. 3) La dinámica del sistema es lenta y los rangos admisibles en las variaciones de las consignas no es muy amplio, con lo que un modelo linealizado de la dinámica del sistema es suficiente para describir adecuadamente la evolución del sistema. 4) Los cambios en las variables de nivel no producen cambios significativos en los valores de régimen permanente de la temperatura y la presión, razón por la cual en el modelo del sistema existe un débil acoplamiento entre las dinámicas de los lazos de nivel y las de los lazos de temperatura y presión. Esto lleva a que sería posible establecer solamente un modelo de dos entradas (flujo de energía y apertura de válvula de flujo de gas) y dos salidas (temperatura y presión) para el diseño de la estrategia de optimización de consignas, dejando por fuera de este esquema a los lazos de nivel, los cuales se implementan mediante lazos PID mediante técnicas de sintonización convencionales. 5) Se asume como disturbio medible, el flujo de alimentación del separador.

A falta de disponibilidad de modelos experimentales sobre un separador real y con las anteriores asunciones, en este trabajo se realiza el siguiente procedimiento para la obtención de los resultados: 1) Simulación del proceso en HYSYS, incluyendo al separador trifásico y la instrumentación requerida para la manipulación de los flujos de interés, donde los lazos de control de nivel de agua y de nivel de petróleo también se implementan en este simulador, 2) Identificación del modelo dinámico del separador tal que sea útil para, donde como entradas manipuladas se tienen el porcentaje de apertura de la válvula de gas $\left(S_{g}\right)$ y el flujo de energía al intercambiador $\left(F_{e}\right)$, como entrada medida no manipulada está el flujo de alimentación del intercambiador $\left(F_{a}\right)$, y como salidas controladas se tienen la temperatura del intercambiador $(T)$ y la presión en la vasija $(p)$, 3) Diseño y simulación en MatLab de la estrategia de control jerárquico de dos fases para el logro de consignas óptimas para la que minimicen $F_{e}$, a pesar de variaciones en $F_{a}$, respetando rangos permitidos para las entradas y salidas.

Para la simulación en HYSYS se realizó el montaje indicado en la Fig. 3. Los datos más relevantes acerca de los parámetros de simulación del separador se resumen en la Tabla 1. Teniendo en cuenta el desempeño del separador para varias condiciones de operación, la Tabla 2 resume los rangos de valores aceptables para las variables manipuladas, y el rango de valores de las variables controladas en régimen permanente, los cuales se establecieron por observación del comportamiento de las variables en simulación. Tales restricciones se usan en los algoritmos de optimización de las ecuaciones (1) a (3), tales que en la búsqueda de valores que optimicen el desempeño económico, no se permita salirse de regiones de operación seguras para el sistema.

Para la identificación se utilizó el toolbox de identificación de sistemas de MatLab ${ }$, generando un conjunto de entradas binarias pseudo aleatorias para las variables de entrada, las cuales se envían a HYSYS en tiempo de simulación mediante un esquema de comunicación ActiveX de MatLab ${ }^{\circledR} 1$

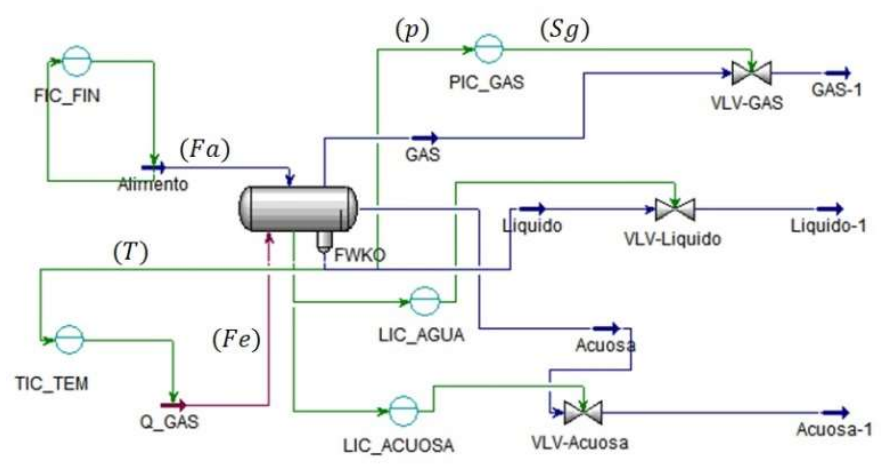

Fig. 3. Montaje del proceso en HYSYS.

Como resultado de la ejecución de la simulación en HYSYS, se registran las salidas durante un tiempo de establecimiento de 100 minutos, con un periodo de muestreo de 600 segundos utilizando el mismo esquema de comunicación. La Fig. 4 muestra una gráfica donde se comparan los registros de salida obtenidos desde el modelo en HYSYS, con los valores predichos por un modelo de espacio de estado lineal de orden 8 obtenido mediante los comandos del Toolbox de identificación de MatLab, representado por las matrices de estado $\boldsymbol{A}_{d}, \boldsymbol{B}_{d}, \boldsymbol{C}_{d}$, y $\boldsymbol{D}_{d}$. El ajuste del modelo logrado muestra un porcentaje de concordancia del $75,76 \%$, con lo que se consideró a dicho modelo como aceptable. 
TABLA I

CARACTERIZACIÓN DEL CRUDO

\begin{tabular}{|l|c|}
\hline $\begin{array}{l}\text { Sutancia }[\mathbf{K g}- \\
\text { mole] }\end{array}$ & Valor \\
\hline Metano & 0,1 \\
\hline Etano & 0,03 \\
\hline Propano & 0,04 \\
\hline i-Butano & 0,08 \\
\hline n-Butano & 0,1 \\
\hline i-Pentano & 0,12 \\
\hline n-Pentano & 0,13 \\
\hline H2O & 0,4 \\
\hline
\end{tabular}

En cuanto al diseño del control jerárquico, se fijó como criterio de optimización la minimización del flujo de energía que se inyecta al intercambiador de calor se puede expresar mediante el criterio de optimización indicado en la ecuación (4).

$$
J_{E}(u)=c_{u}^{T} u^{s s}, c_{u}^{T}=\left[\begin{array}{lll}
1 & 0 & 0
\end{array}\right], u=\left[\begin{array}{lll}
F_{e} & S_{g} & F_{a}
\end{array}\right]^{T}
$$

TABLA II

RANGOS OPERACIONALES DE VARIABLES DE ENTRADA Y SALIDA

\begin{tabular}{|l|c|c|c|}
\hline \multicolumn{1}{|c|}{ Variable } & $\begin{array}{c}\text { Límite } \\
\text { Mínimo }\end{array}$ & $\begin{array}{c}\text { Límite } \\
\text { Máximo }\end{array}$ & Unidades \\
\hline $\begin{array}{l}\text { Flujo } \\
\text { Energía }\end{array}$ & 0 & 3000000 & {$[\mathrm{KJ} / \mathrm{h}]$} \\
\hline $\begin{array}{l}\text { Flujo } \\
\text { Mezcla }\end{array}$ & 0 & 200 & {$[\mathrm{Kg}-\mathrm{mol} / \mathrm{h}]$} \\
\hline Presión & 101.32 & 600 & {$[\mathrm{kPa}]$} \\
\hline Temperatura & 50 & 72 & {$[\mathrm{C}]$} \\
\hline
\end{tabular}

Para el cálculo de la función $\boldsymbol{H}(k)$ requerida en la ecuación (2), desde el modelo lineal identificado se puede calcular la matriz de ganancia de régimen permanente Gss según la ecuación (5).

$$
\boldsymbol{G s s}=\boldsymbol{C}_{d}\left(-\boldsymbol{A}_{d}^{-1} \boldsymbol{B}_{d}\right)
$$

Dado que el modelo es lineal y que el modelo es válido para pequeños incrementos sobre el punto de operación, se cumple la relación de la ecuación (6).

$$
y^{s s}=\boldsymbol{G} \boldsymbol{s} \boldsymbol{s}\left[\begin{array}{c}
F_{e}(k) \\
S_{g}(k) \\
F_{a}(k)
\end{array}\right]=\boldsymbol{H}(k)
$$

De la misma forma, la función $\boldsymbol{F}\left(u^{s s}, \widetilde{w}\right)$ de la ecuación (1) se hace corresponder según (7), donde $u^{s s}$ corresponde al vector de las dos variables manipuladas $F_{e}{ }^{s s}$ y $S_{g}{ }^{s s}$, mientras que el disturbio $\widetilde{w}$ corresponde a la variable medida $F_{a}$.

$$
y^{s s}=\boldsymbol{G s s}\left[\begin{array}{c}
F_{e} s s \\
S_{g} s s \\
F_{a}
\end{array}\right]=\boldsymbol{F}\left(u^{s s}, \widetilde{w}\right)
$$

Los problemas LSSO, SSTO y MPC-DO de las ecuaciones (1), (2) y (3) se pueden resolver en con los algoritmos de optimización de MatLab: fmincon, linprog y $m p c$, respectivamente. El trabajo en [18] utiliza un modelo lineal del sistema para la optimización de un sistema eléctrico, en el cual se utilizó la función fmincon, de Matlab para la implantación del algoritmo de optimización.
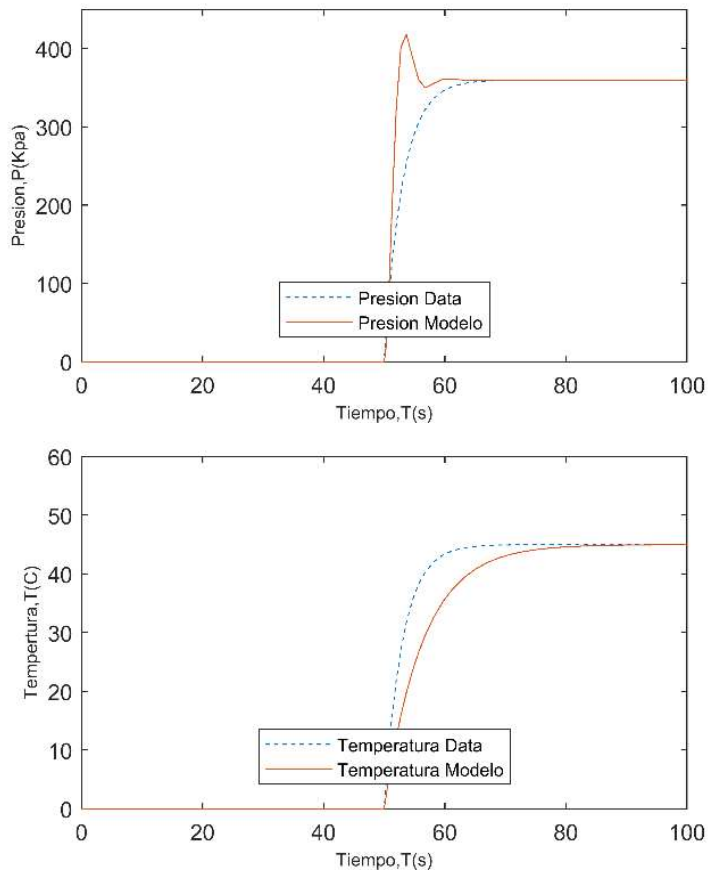

Fig. 4 Comparación de la respuesta del modelo lineal, con la respuesta del modelo en HYSYS.

\section{RESULTADOS Y DISCUSIÓN}

En esta sección se presentan resultados de simulación que permiten evaluar el desempeño económico del control jerárquico en contraste con el esquema PID convencional. Para ello, se plantean dos experiencias de simulación donde se registra el valor al final del tiempo de simulación, de la integral del flujo energético requerido por el separador trifásico. Este valor es un indicador del consumo energético total en ese tiempo.

La primera experiencia se implementa en el entorno HYSYS $\mathrm{y}$ tiene por objeto comparar un esquema PID convencional con el esquema jerárquico de dos fases para dos casos 1) supervisor con solo una capa LSSO (LSSO/PID) y 2) supervisor con capas LSSO y SSTO (LSSO/SSTO/PID). En el caso de esquema convencional, se usaron como consignas fijas $52^{\circ} \mathrm{C}$ en el lazo de temperatura y de 400 psi en el lazo de presión. La segunda experiencia implementa un controlador de bajo nivel tipo MPC en lugar de los lazos PID, pero esta vez solo en el entorno de MATLAB.

En la Fig. 5 a) y b) se observa una comparación de las variables controladas ante las variaciones del flujo de alimentación del separador indicadas en la Fig. 5 c), para los casos PID y LSSO/SSTO/PID de la primera experiencia descrita. Como se observa, los controladores consiguen seguir las referencias a pesar de los cambios en el de flujo de entrada, $\mathrm{y}$ todos logran que los valores se mantengan dentro de los 
rangos indicados en líneas punteadas y consignados en la Tabla 2.

En la Fig. 6 se muestra la comparación de los flujos de energía para los tres casos expuestos en la experiencia uno. Al evaluar la integral del flujo de energía durante los $3 \times 10^{5}$ segundos (correspondientes a 3,47 días), el consumo obtenido para los tres casos es 555442310.7 KJoulios para el caso PID, 386705195.8 KJoulios para el caso LSSO/SSTO/PID, donde se aprecia que éste último esquema de control favorece la reducción del consumo energético total.
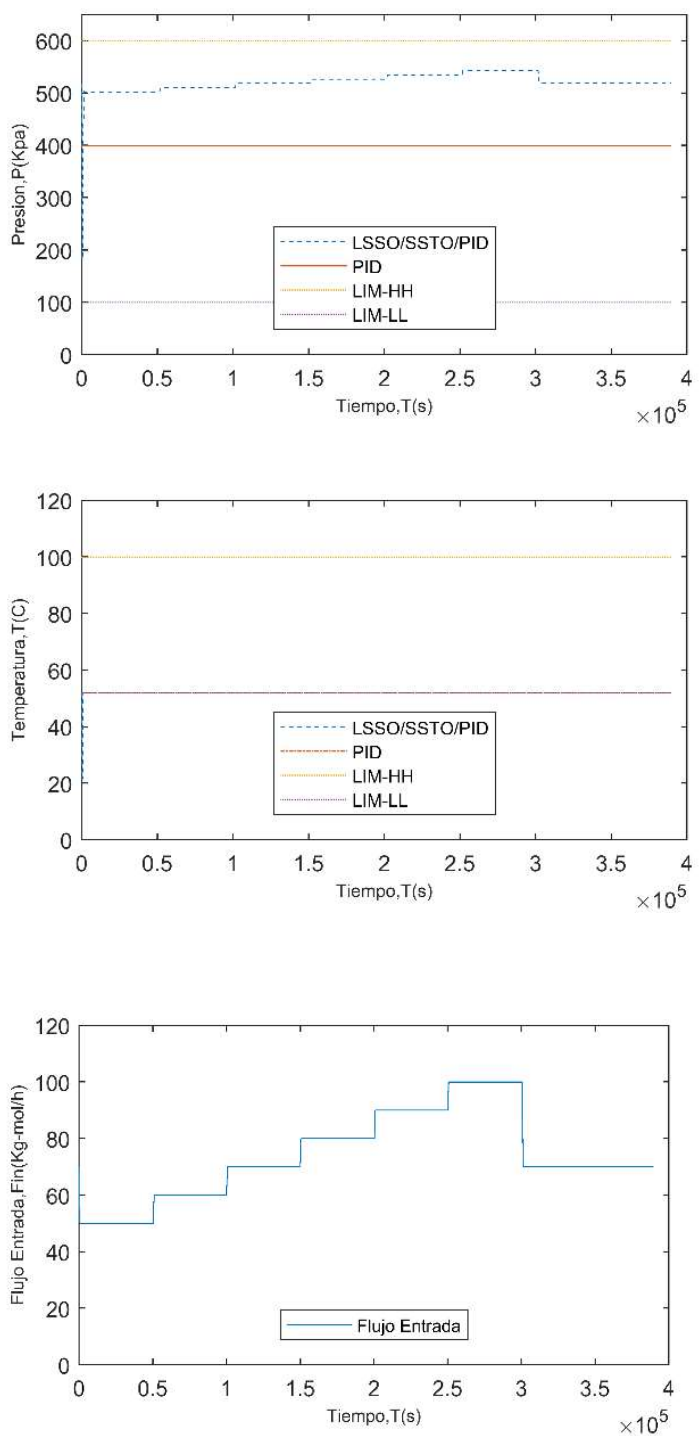

Fig. 5. Resultados de la experiencia uno. a) y b) Comparación de las variables de presión y temperatura para los casos PID, LSSO/SSTO/PID en el entorno HYSYS. c) Variaciones en el flujo de alimentación utilizadas en la simulación.

La segunda experiencia se implementó totalmente en el entorno de MatLab, utilizando el modelo del intercambiador trifásico identificado. Esta experiencia permite comprobar en un entorno de simulación con mayor precisión de simulación dinámica como es MatLab, si los resultados obtenidos en HYSYS también se corroboran en este entorno., a pesar de la simplificación del modelo dinámico empleado. Para esta experiencia, se implementan los esquemas de control 1) PID, 2) LSSO/SSTO/PID y 3) LSSO/SSTO/MPC, utilizando el mismo flujo de alimentación y los mismos rangos de operación utilizados para la experiencia uno.

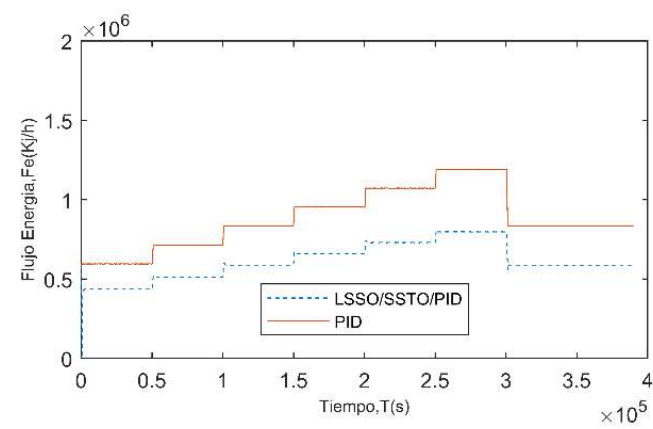

Fig. 6. Comparación de los flujos de energía consumidos por el tratador de calor en los tres esquemas: PID, LSSO/PID y LSSO/SSTO/PID.
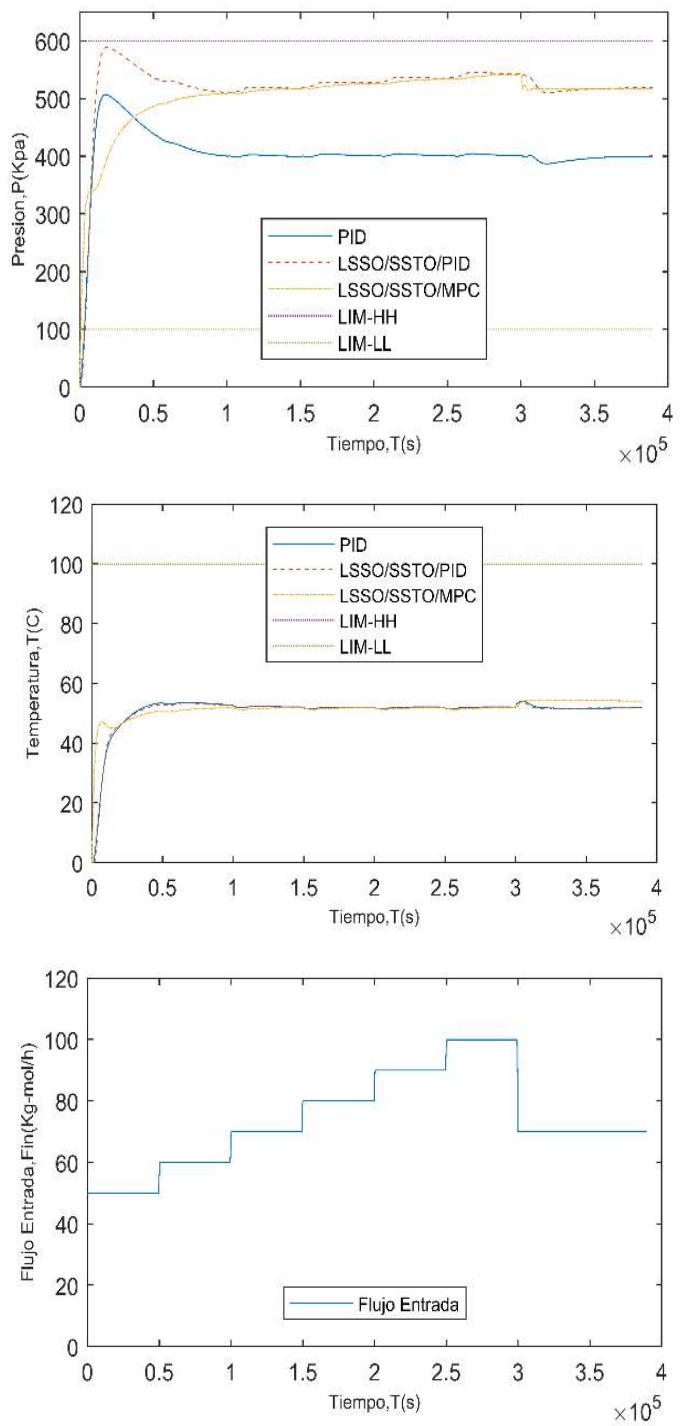

Fig. 7. Resultados de la experiencia dos. a) y b) Comparación de las variables de temperatura y presión para los casos PID, LSSO/SSTO/PID y LSSO/SSTO/MPC en el entorno MatLab. b) Variaciones en el flujo de alimentación utilizadas en la simulación. 
La Fig. 7 muestra el comparativo del comportamiento de las variables controladas frente a los cambios de flujo de alimentación, mientras que la Fig. 8 muestra el comparativo de los correspondientes flujos de energía consumidos por el tratador de calor para los tres esquemas implementados.

En esta experiencia, al integrar el flujo de energía consumida durante el tiempo de simulación se obtienen los consumos de energía total de 910453665.8 KJoulios para el caso PID, 898244510 KJoulios para el caso LSSO/SSTO/PID y 887459530.7 KJoulios para el caso LSSO/SSTO/MPC donde se aprecia que el esquema de control LSSO/SSTO/MPC es el que más favorece la reducción del consumo energético total.

Observando los comportamientos de las variables controladas tanto en la Fig. 5 como en la Fig. 7 se concluye que en el esquema convencional tipo PID donde las consignas están fijas en independencia del flujo de alimentación, el balance energético lleva a que a mayor flujo de alimentación, mayor sea el consumo de energía requerido para mantener tanto la consigna de temperatura como la de presión.

A fin de minimizar el consumo energético se minimiza la consigna de temperatura, razón por la cual ésta siempre se establece en el menor valor permitido. Sin embargo, el ahorro logrado se explica por la posibilidad de cambiar la consigna de presión en función de los cambios de alimentación, tal que el balance energético se cumpla en regiones de menor consumo de energía del tratador de calor.

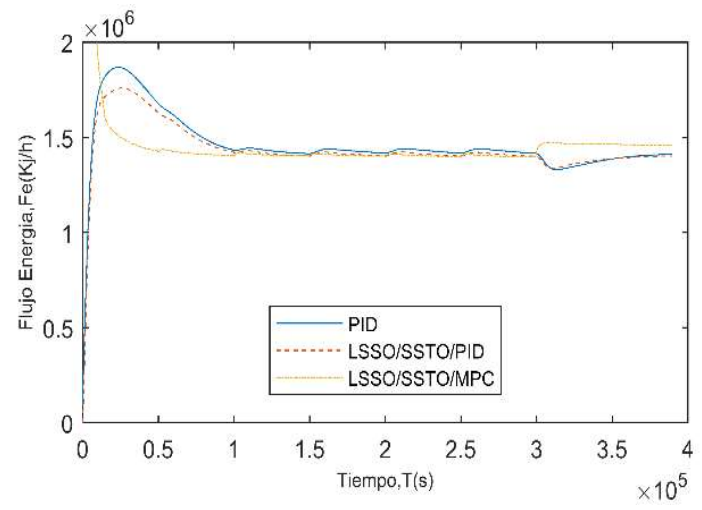

Fig. 8. Comparación de los flujos de energía consumidos por el tratador de calor en el entorno Matlab para los tres esquemas: PID, LSSO/SSTO/PID, y $\mathrm{LSSO} / \mathrm{SSTO} / \mathrm{MPC}$.

\section{CONCLUSIONES}

Se ha mostrado que un es viable utilizar un esquema de control jerárquico de dos fases para el logro del objetivo de mantener la presión y temperatura de un separador trifásico con tratamiento de calor dentro de rangos de operación definidos por la seguridad de la planta, al tiempo que se logre un beneficio en la minimización del consumo energético de la planta. Esta conclusión se sustenta al comparar el consumo total de energía durante el tiempo de simulación, frente a un esquema tradicional de consignas fijas con lazos PID. Trabajos futuros deben ir encaminados hacia una simulación más realista del tren de tratamiento de crudo, donde se analicen parámetros de interés en cuanto al rendimiento de la separación lograda en el tren de tratamiento, como función de los distintos puntos de operación para las variables a controlar, y también hacia una comprobación experimental de la técnica propuesta sobre una planta de tratamiento real. Para ello, es necesario contar con instrumentación adecuada que permita implementar algoritmos de optimización en tiempo real y estrategias de control MPC.

\section{REFERENCIAS}

[1] Arnold.K, Stewart.M, "Chapter 6 - Crude Oil Treating Systems", Surface Production Operations: Design of Oil-Handling Systems and Facilities (Second Edition), Gulf Professional Publishing, pp. 160193, 1999. https://doi.org/10.1016/B978-0-88415-821-9.X5000-3.

[2] Filgueiras.N, "Modelagem análise e controle de um processo de separacao óleo/água",tesis, Universidade Federal do Rio de Janeiro, 2005.

[3] P. R. C. Mendes, J. E. Normey-Rico, A. Plucenio, and R. L. Carvalho, "Disturbance estimator based nonlinear MPC of a three phase separator", IFAC, vol 45,pp 101-106, 2012, https://doi.org/10.3182/20120710-4-SG-2026.00060.

[4] Mendes P. R, Carvalho R. L and Normey-Rico J. "Nonlinear predictive control applied to load oscillations damping in a three phese separator", in Rio Pipeline 2011.

[5] León O. G, Sosa Y. R. and Álvarez C. M, “Alternativas de mejoras energéticas en la planta de tratamiento de crudos de puerto escondido", Rev. Ingeneiría Energética, vol. XXXVIII, pp. 115123,2017,DOI:http://scielo.sld.cu/scielo.php?script=sci_arttext\&pid $=$ S1815-59012017000200006.

[6] Tatjewski, P. "Advanced control and on-line process optimization in multilayer structures", IFAC, vol. 11, no. PART 1, pp. 13-26, 2007,https://doi.org/10.1016/j.arcontrol.2008.03.003.

[7] Pan H, Zhong W and Wang Z, "An on-line constraint softening strategy to guarantee the feasibility of dynamic controller in doublelayered MPC", Chinese Journal of Chemical Engineering,Volume 25,pp 1805-1811, 2017, https://doi.org/10.1016/j.cjche.2017.06.010.

[8] Aspentech, "Aspen HYSYS Dynamics", [Online]. Available: https://www.aspentech.com/en/products/pages/aspen-hysysdynamics. [Accessed: 15-Nov-2018].

[9] Famisa .R. B, "HYSYS Modelling of a Horizontal Three-Phase Subsea Separator",Tesis, Norwegian University of Science and Technology , Department of Chemical Engineering,Norway, 2016.

[10] Stewart.M and Arnold .K, "Chapter 1 - Crude Oil Treating Systems", Emuls. Oil Treat. Equip, pp. 1-80, 2009, https://doi.org/10.1016/B978-0-7506-8970-0.00001-3.

[11] Sayda A. F. and Taylor J. H, "Modeling and control of three-phase gravity separators in oil production facilities", Proc. Am. Control Conf., pp. 4847-4853, 2007, DOI: 10.1109/ACC.2007.4282265.

[12] Kharoua.N, Khezzar.L, and Saadawi. H, "CFD Modelling of a Horizontal Three-Phase Separator: A Population Balance Approach", Am. J. fluid Dyn., vol. 3, no. 4, pp. 101-118, 2013, DOI: 10.5923/j.ajfd.20130304.03.

[13] Jekel.T. B, Reindl D. T, and Fisher. J. M, "Gravity Separator Fundamentals and Design", IIAR 2001 Ammonia Refrigeration Convention \& Exhibition, Long Beach,CA, p. 23,2001.

[14] Santos .M. N, Caio. P. R, Mariz .M, Pedro .S, A. de M. Arioston, and L. O. A. Rojas, "Modelagem e simulação de um separador trifásico de petróleo autores", in 9o Congresso Brasileiro De Pesquisa E Desenvolvimento Em Petróleo E Gás, vol. 25, no. 4,2013 , pp. 628638.

[15] Pèrez .N.M "Propuesta Metodológica Para el Diseño y Operación de Instalaciones de Separación y Procesamiento de Crudo ", tesis maestria, Universiada Nacional de Colombia, 2017.

[16] Cavalcanti .G, "Design and Analysis of Multivariable Predictive Control",tesis, University of Florida, 2001.

[17] Figueiredo. A. I. L. and Sotomayor A. Z, "Aspectos de controle de um processo de separação água-óleo-gás", VIII Congresso Brasileiro de Engenharia Química em Iniciação Científica, p. 8, 2009.

[18] Méndez D. E , Sánchez J. y Alzate R, "Control óptimo conmutado de un convertidor CC-CC", Entre Ciencia e Ingeniería, Año 10 No. 19, página 16-21. DOI: http://dx.doi.org/10.31908/19098367.2793. 
Sander Sánchez Reyes. Nació en Cajibío, Cauca, el 21 de febrero de 1978. En el año 2010 Se graduó en la Universidad del Cauca de Ingeniero en Automática Industrial. Ejerció profesionalmente en el año 2012 en la compañía OMNICON SA como Ingeniero de Proyectos de Automatización, posteriormente en al año 2014 ejerció en la compañía PETRO TIGER el cargo de Ingeniero de Mantenimiento Mayor, en el año 2016 ejerció el cargo de Ingeniero de Automatización en la compañía Massy Energy y desde el año 2017 se desempeña como Ingeniero de Control en la empresa Confipetrol en Campo Rubiales Meta.

ORCID: https://orcid.org/0000-0002-4306-7876.

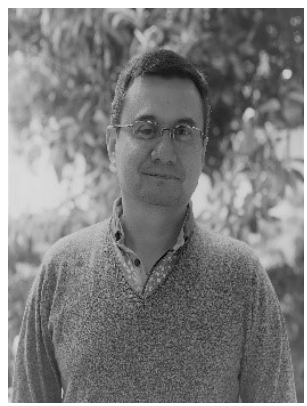

Carlos Alberto Gaviria. Nació en Popayán, Cauca, el 2 de diciembre de 1969. Ingeniero en Electrónica y Telecomunicaciones de la Universidad del Cauca en 1994, obtuvo su título como PhD. en Automatización avanzada y Robótica en la Universidad Politécnica de Catalunya en el año 2004. Ha sido profesor en la Universidad del Cauca desde el año 1997, donde ahora es profesor titular. Ha sido director del grupo de investigación en Automática Industrial de la Universidad del Cauca y sus áreas de investigación son la teoría de control, y las aplicaciones de la automática en la salud.

ORCID: https://orcid.org/0000-0002-9773-7660. 